\title{
LOS CORRALES DE VECINOS EN LA SEVILLA DEL SIGLO DE ORO
}

\section{COURTYARD TENEMENT HOUSING IN $16^{\mathrm{TH}}$ CENTURY SEVILLE}

\author{
MARÍA NúÑEZ GonZÁLEZ \\ Grupo de investigación HUM976: Expregrafica. \\ Lugar, Arquitectura y Dibujo. España \\ ORCID: 0000-0002-0022-7921 \\ mngsevilla@gmail.com
}

\begin{abstract}
Este artículo recoge el análisis de una de las tipologías arquitectónicas residenciales modestas más reconocidas y menos analizadas en el siglo XVI en Sevilla, los corrales de vecinos. Además, se debe resaltar la existencia de numerosos corrales repartidos por toda la ciudad en el Siglo de Oro, y se ha reflejado el análisis de su ubicación, nombres, construcción y espacios: se han localizado sobre el plano de la ciudad en dicho periodo, se han dibujado los inmuebles descritos en los apeos literarios formulando hipótesis en planta para facilitar la comprensión y análisis arquitectónico y, por último, se han caracterizado y analizado los espacios, su construcción y dotaciones, todo ello a partir de los apeos y de los dibujos realizados.

Palabras clave: arquitectura; corrales de vecinos; Sevilla; siglo XVI; apeos.
\end{abstract}

This paper contains the analysis of one of the most well-known and modest architectural types of residence in the $16^{\text {th }}$ century Seville: courtyard tenement housing. Besides, it should be highlighted the existence of a great number of them distributed inside the walls of the city in the Golden Century, and an analysis of location, names, construction and spaces have been studied based in literary surveys and drawings: first there have been identified twenty seven cases of study later located in an elaborated map of city; secondly drawings based in literary surveys have been made to facilitate the architectural analysis and the specifications of this residential type; and finally, the courtyard tenement housing -spaces, construction elements, water supplies, sanitation, etc.- has been characterized and fully studied.

Key words: architecture; courtyard tenement housing; Seville; $16^{\text {th }}$ century; surveys.

\section{APROXIMACIÓN TIPOLÓGICA DE LOS CORRALES}

Fue Joaquín Hazañas el primero que intentara en su día una definición del corral de vecinos: "las palabras patio y corral son sinónimas en nuestra lengua [...], por esa misma razón llamamos en Sevilla a la casa de vecindad corral de vecinos, 
porque todos ellos fueron, y lo son los que aún subsisten, un gran patio, y nada más que eso, rodeados de habitaciones"1. A pesar de esta sobria contundencia conceptual, con los datos disponibles se podría decir, en efecto, y someramente, que esta tipología residencial se configuraba en Sevilla mediante la construcción de una casa en un solar interior -huerta o compás- por medio de una crujía de habitaciones que se adosaban a los linderos. No obstante, como en adelante se verá, caben algunas matizaciones y ampliaciones a esa primera aproximación.

Antonio Collantes de Terán dedica a los corrales unas páginas en su libro sobre la Sevilla en la Baja Edad Media. Cita la cantidad de ellos por collaciones y barrios ${ }^{2}$, llegando a contar en torno a cincuenta corrales durante el siglo XV. Aunque no se detiene en analizar en profundidad esta tipología-solo estudia dos casos-, sí distingue entre los corrales de vecinos propiamente dichos de una o dos plantas - con una casa en la entrada- y la casa común que era habitada por varias familias.

Un análisis más pormenorizado de esta particular solución habitacional fue realizado en 1978 por Antonio Barrionuevo y Francisco Torres en su publicación En torno a la casa sevillana ${ }^{3}$, que la describían compuesta por al menos una crujía de dos plantas y la presencia de galerías en la superior. Las características de la crujía - establecidas en la relación entre construcción de muros y aplicación de la carpintería o cerrajería en las galerías-y las del patio, singularizaban cada corral. Años más tarde, Barrionuevo volvería a retomar el tema mediante su participación en el seminario La Casa y el Lugar, organizado por Gonzalo Díaz Recasens en $1999^{4}$.

Francisco Morales Padrón por su parte, en Los corrales de vecinos de Sevilla (informe para su estudio), publicado en $1991^{5}$, pone de manifiesto las características de esta tipología arquitectónica tras el análisis de más de cincuenta corrales existentes en ese momento en Sevilla y afirma que el corral o patio sevillano no alberga una población perteneciente a la llamada "cultura de la pobreza", ya que su morada peculiar se encuentra dispersada por toda la ciudad y no concentrada en una zona concreta. Esta última idea, según lo analizado en este artículo, se

${ }^{1}$ HAZAÑAS Y LA RÚA, Joaquín: Algunas consideraciones sobre la casa sevillana. En la inauguración de las Conferencias sobre asuntos sevillanos. Sevilla, 1928 (ed. 1989).

2 COLlANTES DE TERÁN, Antonio: Sevilla en la Baja Edad Media. Sevilla, 1984, pp. 123-124.

${ }^{3}$ BARRIONUEVO, Antonio y TORRES, Francisco: "En torno a la casa sevillana", 2C. Construcción de la ciudad, 11, 1978, pp. 16-22.

${ }^{4}$ BARRIONUEVO FERRER, Antonio: "La arquitectura del patio desde Sevilla", en DÍAZ RECASÉNS, Gonzalo (dir.): La Casa Meridional. Correspondencias. Sevilla, 2001, pp. 123-138.

5 MORALES PADRÓN, Francisco (dir. red.): Los corrales de vecinos de Sevilla (informe para su estudio). $2^{\mathrm{a}}$ ed. Sevilla, 1991. 
puede trasponer al estudio de los corrales en el siglo XVI. Para Morales Padrón, el corral no es una solución de emergencia, tipo hongo, sino que es una respuesta arquitectónica especial que posee rasgos comunes con sus parientes tipológicos canarios y americanos ${ }^{6}$.

Más recientemente, otros autores han investigado sobre los corrales en la actualidad. Según su localización, son Fernández Salinas ${ }^{7}$ y Morgado Giraldo en Sevilla, García Gómez ${ }^{9}$ en Málaga, y Martínez Portilla ${ }^{10}$ en Cádiz. Víctor Fernández Salinas documentó más de cuatrocientos corrales, casas de vecindad y corralones en 1994, cifra que se rebajó a cerca de la mitad en siete años. Este dato da una referencia en cuanto a la importancia de esta tipología arquitectónica y de su evolución en la ciudad de Sevilla desde el siglo XVI hasta fecha reciente.

Conociendo estas aportaciones al estudio de los corrales de la Sevilla contemporánea, se ha regresado al origen de estos, es decir, al siglo que nos ocupa, heredero a su vez de la Sevilla medieval, en la que no cabe duda de que ya existían también como tales, haciendo uso de la información que nos ofrecen las fuentes históricas consultadas ${ }^{11}$. A partir de ellas se analizarán los corrales del siglo XVI y se establecerán las conclusiones sobre esta tipología, con el fin de poder

${ }^{6}$ LEWIS, Oscar: "La cultura de la vecindad en la ciudad de México", Ciencias Políticas y Sociales, 17, 1959, pp. 360-372; y SEBRELI, Juan José: Buenos Aires, vida cotidiana y alienación. Buenos Aires, 1965.

${ }^{7}$ FERNÁNDEZ SALINAS, Víctor: "La vivienda modesta y patrimonio cultural: Los corrales y patios de vecindad en el conjunto histórico de Sevilla", Scripta Nova. Revista electrónica de geografía y ciencias sociales, 146(070), 2003: http://www.ub.es/geocrit/ sn/sn-146(070).htm.

${ }^{8}$ MORGADO GIRALDO, Ricardo: Los Corrales y Patios de Vecinos de Triana. Sevilla, 1994, p. 80.

${ }^{9}$ GARCÍA GÓMEZ, Francisco: "La desaparición de una tipología doméstica: los últimos corralones malagueños", Boletín de arte, 18, 1997, pp. 171-198.

${ }^{10}$ MARTÍNEZ PORTILLA, Isabel María: "En Torno al Patio. Breve Acercamiento a las Casas de Vecinos del Barrio de la Viña, Cadiz", Demófilo, 24, 1997, pp. 229-244.

${ }^{11}$ NÚÑ̃Z GONZÁLEZ, María: La casa sevillana del siglo XVI en la collación de San Salvador: dibujo y estudio de tipologías. Sevilla, 2012; NÚÑEZ GONZÁLEZ, María: Casas, corrales, mesones y tiendas en la Sevilla del siglo XVI. Arquitectura, dibujo y léxico de alarifes. Tesis doctoral, Universidad de Sevilla, 2017; NÚÑEZ GONZÁLEZ, María: "Metodología para el estudio, dibujo y localización de casas sevillanas de los siglos XVI y XVII", Atrio. Revista de Historia del Arte, 22, 2016, pp. 72-85; NÚÑ̃Z GONZÁLEZ, María: "The Role of Drawing and Master Alarifes in the Study of the Sixteenth and Seventeenth Centuries Sevillian Housing from Graphical and Literary Documents", Graphic Imprints, 2019, pp. 685-698: https://doi.org/10.1007/978-3-319-93749-6_55; y NÚÑEZ GONZÁLEZ, María: "Casas, corrales, mesones y tiendas en la Sevilla del siglo XVI. Arquitectura, dibujo y léxico de alarifes", en Actas del I Congreso internacional de Doctorados de Arquitectura. IDA: Advanced doctoral research in architecture. Sevilla, 2017, pp. 907-930. 
comparar las características de las que se partían con las arrojadas tras el análisis realizado.

\section{REFERENCIA DOCUMENTAL, LOCALIZACIÓN Y ANÁLISIS URBANO}

Las fuentes utilizadas para la localización de los corrales de vecindad en Sevilla en el siglo XVI han sido los libros de apeos del cabildo, la fábrica, el comunal y los de los hospitales de las Cinco Llagas, el Cardenal, las Bubas, Amor de Dios y Espíritu Santo, además de otros documentos del Archivo de Protocolos Notariales de Sevilla ${ }^{12}$.

La información que se ha podido recoger sobre los corrales es la que se ofrece en la tabla 1, donde se identifican por su nombre -a veces llevan el de su propietario o administrador y en otros casos el conocido popularmente-, su situación en el casco urbano -collación y calle- y la propiedad de estos. Cuando estaban en manos de las grandes instituciones eclesiásticas y asistenciales, estos inmuebles eran arrendados de por vida a particulares, cuyas referencias aparecen en los apeos citados; y en el supuesto de una propiedad no institucional, la práctica común era el arrendamiento por años o por meses, como si se tratase de una casa.

Tabla 1. Listado y tabla de datos generales sobre los corrales de vecindad documentados

\begin{tabular}{|c|c|c|c|c|}
\hline $\mathbf{N}$ & Nombre & Collación & Situación & Propietario \\
\hline 1 & $\begin{array}{l}\text { De Salvador } \\
\text { García }\end{array}$ & (Extramuros) & Fuera de la Puerta de la Macarena & $\begin{array}{l}\text { Salvador Gar- } \\
\text { cía, clérigo }\end{array}$ \\
\hline 2 & De las Cañas & $\begin{array}{l}\text { La } \\
\text { Magdalena }\end{array}$ & Calle de los Ángeles & $\begin{array}{l}\text { Hospital del } \\
\text { Cardenal }\end{array}$ \\
\hline 3 & $\begin{array}{l}\text { En la barrera } \\
\text { de Alvar } \\
\text { Negro }\end{array}$ & $\begin{array}{l}\text { Omnium } \\
\text { Sanctorum }\end{array}$ & En la barrera de Alvar Negro & $\begin{array}{l}\text { Rodrigo } \\
\text { Franco, mer- } \\
\text { cader, las dejó } \\
\text { a la Fábrica }\end{array}$ \\
\hline 4 & De Gallinas & $\begin{array}{l}\text { Omnium } \\
\text { Sanctorum }\end{array}$ & $\begin{array}{l}\text { Las fuentes no lo indican, pero } \\
\text { existe una calle con este nombre, } \\
\text { así que puede que se localizase allí }\end{array}$ & - \\
\hline 5 & $\begin{array}{l}\text { De la calle } \\
\text { de Ciegos }\end{array}$ & $\begin{array}{l}\text { Omnium } \\
\text { Sanctorum }\end{array}$ & Calle Ciegos & $\begin{array}{l}\text { Hospital } \\
\text { Amor de Dios }\end{array}$ \\
\hline
\end{tabular}

12 ACS (Archivo de la catedral de Sevilla), Mesa Capitular, sección Cabildo, lib. 9163, 1542; ACS, sección Fábrica, lib. 9717, 1543; ADPS (Archivo de la Diputación Provincial de Sevilla), hospital de las Bubas, lib. 3bis, 1585; ADPS, hospital de las Cinco Llagas, lib. 1, 1541-1545; y ADPS, hospital del Cardenal. 


\begin{tabular}{|c|c|c|c|c|}
\hline $\mathbf{N}$ & Nombre & Collación & Situación & Propietario \\
\hline 6 & $\begin{array}{l}\text { De la calle } \\
\text { del Peral }\end{array}$ & $\begin{array}{l}\text { Omnium } \\
\text { Sanctorum }\end{array}$ & Calle Peral & $\begin{array}{l}\text { Hospital } \\
\text { Amor de Dios }\end{array}$ \\
\hline 7 & $\begin{array}{l}\text { De las Pal- } \\
\text { mas }\end{array}$ & $\begin{array}{l}\text { San Barto- } \\
\text { lomé }\end{array}$ & $\begin{array}{l}\text { Linda con unas casas que tiene el } \\
\text { licenciado Luis de Coronado y su } \\
\text { mujer Leonor de Bustamante en la } \\
\text { collación de San Bartolomé en la } \\
\text { plazuela que dicen del Tinte de las } \\
\text { Parras y que lindan por las espal- } \\
\text { das con el corral }\end{array}$ & - \\
\hline 8 & $\begin{array}{l}\text { En San Ber- } \\
\text { nardo }\end{array}$ & San Bernardo & $\begin{array}{l}\text { En San Bernardo, junto al Taga- } \\
\text { rete }\end{array}$ & $\begin{array}{l}\text { Doctor An- } \\
\text { drés Çamudio } \\
\text { de Alfaro } \\
\end{array}$ \\
\hline 9 & Del Moro & $\begin{array}{l}\text { San Marcos/ } \\
\text { San Román }\end{array}$ & $\begin{array}{l}\text { Calle que va de San Román a San } \\
\text { Marcos (actual calle Socorro) }\end{array}$ & $\begin{array}{l}\text { Matrimonio } \\
\text { vasco Ortuño } \\
\text { de Bilbao la } \\
\text { Vieja y Ma- } \\
\text { ría Ochoa su } \\
\text { mujer }\end{array}$ \\
\hline 10 & $\begin{array}{l}\text { De Juan } \\
\text { Guerra }\end{array}$ & San Martín & En la Costanilla de San Martín & $\begin{array}{l}\text { Juan Guerra, } \\
\text { mercader de } \\
\text { azúcar } \\
\end{array}$ \\
\hline 11 & De Toqueros & San Nicolás & - & - \\
\hline 12 & $\begin{array}{l}\text { De los Trom- } \\
\text { peros }\end{array}$ & San Nicolás & $\begin{array}{l}\text { Calle Vírgenes (en la collación } \\
\text { de San Isidro, como venimos del } \\
\text { Candilejo para el Corral de los } \\
\text { Tromperos) }\end{array}$ & - \\
\hline 13 & $\begin{array}{l}\text { De Martín } \\
\text { López }\end{array}$ & San Salvador & $\begin{array}{l}\text { Calle de la Ballestilla (como veni- } \\
\text { mos de la dicha Ballestilla a la de- } \\
\text { recha) }\end{array}$ & $\begin{array}{l}\text { Martin } \\
\text { López, chapi- } \\
\text { nero }\end{array}$ \\
\hline 14 & De las Vacas & San Salvador & $\begin{array}{l}\text { Ollería y Carnicería (como veni- } \\
\text { mos de la Ollería para ir al hos- } \\
\text { pital de los Carniceros a mano } \\
\text { derecha pasada la calle que va al } \\
\text { Corral de las Vacas) }\end{array}$ & - \\
\hline 15 & $\begin{array}{l}\text { De la Mo- } \\
\text { rena }\end{array}$ & San Salvador & $\begin{array}{l}\text { Calle que va del barrio de Don Pe- } \\
\text { dro Ponce al Mesón de la Castaña } \\
\text { (calle que va a la Vinatería) }\end{array}$ & $\begin{array}{l}\text { Hospital de } \\
\text { las Cinco } \\
\text { Llagas }\end{array}$ \\
\hline 16 & $\begin{array}{l}\text { De la Balles- } \\
\text { tilla }\end{array}$ & San Salvador & A la Ballestilla & $\begin{array}{l}\text { Hospital de } \\
\text { las Bubas }\end{array}$ \\
\hline
\end{tabular}




\begin{tabular}{|c|c|c|c|c|}
\hline $\mathbf{N}$ & Nombre & Collación & Situación & Propietario \\
\hline 17 & $\begin{array}{l}\text { De las Mo- } \\
\text { zas }\end{array}$ & San Salvador & $\begin{array}{l}\text { En una callejuela sin salida que } \\
\text { llaman la calleja del Mesón de las } \\
\text { Mozas en la calle de la Sierpes }\end{array}$ & $\begin{array}{l}\text { Malgarida } \\
\text { Hernández, } \\
\text { viuda de Fran- } \\
\text { cisco López }\end{array}$ \\
\hline 18 & $\begin{array}{l}\text { De Mara- } \\
\text { ñuelo }\end{array}$ & San Salvador & $\begin{array}{l}\text { No dice. Recibe el nombre de Co- } \\
\text { rral de Moraderas (moradas) }\end{array}$ & $\begin{array}{l}\text { Doña Ana Se- } \\
\text { garra (hasta } \\
1544 \text { ) / Don } \\
\text { Juan Ponce de } \\
\text { León }\end{array}$ \\
\hline 19 & Del Agua & $\begin{array}{l}\text { Santa Cata- } \\
\text { lina }\end{array}$ & $\begin{array}{l}\text { Plaza del Carbón o Duque de Ar- } \\
\text { cos }\end{array}$ & $\begin{array}{l}\text { Hospital de } \\
\text { las Cinco Lla- } \\
\text { gas }\end{array}$ \\
\hline 20 & $\begin{array}{l}\text { De la calle } \\
\text { Sol }\end{array}$ & Santa Lucía & Calle Sol, frontero del adarve & $\begin{array}{l}\text { Hospital del } \\
\text { Cardenal }\end{array}$ \\
\hline 21 & $\begin{array}{l}\text { De la calle de } \\
\text { Santa María } \\
\text { la Blanca }\end{array}$ & $\begin{array}{l}\text { Santa María } \\
\text { la Blanca }\end{array}$ & $\begin{array}{l}\text { En la puerta de la Carne en la ca- } \\
\text { lle de la iglesia de Santa María la } \\
\text { Blanca }\end{array}$ & $\begin{array}{l}\text { Doña Fran- } \\
\text { cisca Rigón, } \\
\text { vende el co- } \\
\text { rral }\end{array}$ \\
\hline 22 & $\begin{array}{l}\text { De don Pe- } \\
\text { dro Suárez }\end{array}$ & $\begin{array}{l}\text { Santa María } \\
\text { la Mayor }\end{array}$ & $\begin{array}{l}\text { Calle de Rosas, en una barrera sin } \\
\text { salida }\end{array}$ & $\begin{array}{l}\text { Pedro Suárez } \\
\text { de Castilla }\end{array}$ \\
\hline 23 & $\begin{array}{l}\text { De San Her- } \\
\text { menegildo }\end{array}$ & $\begin{array}{l}\text { Santa María } \\
\text { la Mayor }\end{array}$ & En el Compás de la Mancebía & $\begin{array}{l}\text { Hospital del } \\
\text { Cardenal }\end{array}$ \\
\hline 24 & Del Conde & Santiago & Calle Santiago & - \\
\hline 25 & $\begin{array}{l}\text { De la calle } \\
\text { del Conde }\end{array}$ & Santiago & $\begin{array}{l}\text { Calle del Conde. Barrio de San- } \\
\text { tiago a la muralla, que lindan con } \\
\text { el jardín y casas del duque de Ve- } \\
\text { raguas }\end{array}$ & $\begin{array}{l}\text { Don Juan de } \\
\text { Vargas y So- } \\
\text { tomayor, } 24 \\
\text { de Sevilla. }\end{array}$ \\
\hline 26 & $\begin{array}{l}\text { De la calle } \\
\text { de la Cava }\end{array}$ & Triana & $\begin{array}{l}\text { Calle de la Cava (o también calle } \\
\text { Nueva) }\end{array}$ & $\begin{array}{l}\text { Matrimonio } \\
\text { vasco Ortuño } \\
\text { de Bilbao la } \\
\text { Vieja y María } \\
\text { Ochoa }\end{array}$ \\
\hline 27 & De la Parra & Triana & Calle de Santo Domingo & - \\
\hline 28 & $\begin{array}{l}\text { De los } \\
\text { Olmos }\end{array}$ & - - & - & - \\
\hline
\end{tabular}


A partir de las fuentes citadas, se han documentado veintisiete ${ }^{13}$ corrales de $^{-}$ vecinos por toda la ciudad, tanto en el interior del recinto amurallado como extramuros, aunque se sospecha que su número sería mucho mayor, especialmente porque cobijaban a personas de condición humilde que no tenían capacidad adquisitiva como para poder pagar el alquiler de una casa por pequeña que esta fuese. La collación que más corrales poseía, según estos datos, era la de San Salvador, con seis, le seguía Omnium Sanctorum con cuatro y las de Santa María la Mayor, Santiago, San Nicolás y Triana con dos respectivamente. En las de la Magdalena, San Bartolomé, San Bernardo, San Marcos (San Román), San Martín, Santa Catalina, Santa Lucía, Santa María la Blanca y extramuros (la Macarena) solo se tiene constancia de la existencia de un corral documentado respectivamente.

Analizando la localización de los corrales (Figura 1), se concluye que esta tipología estaba extendida por toda la ciudad, y salvo en el caso de la collación de San Salvador, en la que se encontraban seis corrales, no se concentraba en una única zona de la ciudad. Además, se construyeron tanto intramuros como extramuros, ya que aparecen corrales en Triana, la puerta de la Macarena y San Bernardo.

El hecho de que estuviera extendida por la ciudad puede hacer pensar que no eran edificios residenciales que acogieran grupos marginales, sino que se trataba de una solución habitacional mínima de origen humilde y que fue el resultado de la colmatación de los solares vacíos por las familias y los trabajadores que emigraban a la ciudad en su época de máxima expansión económica y cultural.

En relación con la manzana, debido a su génesis, los corrales podían situarse en una posición centrada, rellenando el centro de esta o también podían ocupar una parte importante de ella. En cuanto al acceso a los mismos, este solía ser mediante un adarve o callejón a través de una casapuerta o entrada arqueada que daba acceso al patio que articulaba todo el conjunto. Por otro lado, ejemplos destacables del segundo caso, en los que el corral ocupaba una unidad dentro una manzana urbana, eran el corral del Conde en la calle Santiago y el de las Cañas en la calle de los Ángeles (actual Méndez Núñez).

\section{SELECCIÓN, ANÁLISIS Y CARACTERÍSTICAS}

Para poder estudiar las características de esta tipología residencial sevillana se han analizado nueve corrales de los que se posee su apeo o dibujo (Tabla 2), y que arrojan información sobre su situación, superficie, organización espacial, estancias e instalaciones.

${ }^{13}$ No se ha contabilizado el corral de los Olmos, porque podría tratarse de las antiguas Casas Consistoriales de Sevilla, y no de un corral de vecinos al uso. 
Tabla 2. Nombre, situación y referencia documental de los corrales analizados.

\begin{tabular}{|l|l|l|l|l|}
\hline $\mathbf{N}$ & \multicolumn{1}{|c|}{ Nombre } & \multicolumn{1}{|c|}{ Collación } & \multicolumn{1}{|c|}{ Situación } & Ref. documental \\
\hline 2 & De las Cañas & La Magdalena & Situado & k136 \\
\hline 3 & $\begin{array}{l}\text { En la barrera de Alvar } \\
\text { Negro }\end{array}$ & Omnium Sanctorum & Situado & f523r \\
\hline 5 & De la calle de Ciegos & Omnium Sanctorum & Situado & a107 \\
\hline 6 & De la calle del Peral & Omnium Sanctorum & Situado & a115 \\
\hline 15 & De la Morena & San Salvador & No situado & p29r \\
\hline 16 & De la Ballestilla & San Salvador & Situado & b10v \\
\hline 17 & De las Mozas & San Salvador & Situado & $\begin{array}{l}\text { AHPSPN, le- } \\
\text { gajo 16740, of. } \\
\text { fol. escrit. 1590, }\end{array}$ \\
\hline 20 & De la calle Sol & Santa Lucía & Situado & k102 \\
\hline 23 & De San Hermenegildo & Santa María la Mayor & No situado & k31 \\
\hline
\end{tabular}

Antes de pasar a analizar las características tipológicas de estos inmuebles se debe puntualizar que las descripciones recogidas en los apeos son, en general, poco detalladas, y en algunos casos incluso faltan medidas de los patios y las estancias, o dan dimensiones globales que no permiten dar una idea de la ocupación o la edificabilidad que poseía la parcela ${ }^{14}$. A pesar de estas dificultades, se han podido analizar y sacar unas primeras conclusiones sobre esta tipología residencial que marcan el origen para futuras investigaciones.

\section{Ocupación de la manzana y superficies}

Como ya se ha adelantado anteriormente, la relación de los corrales dentro de la manzana urbana podía realizarse de diversas formas, dependiendo de su extensión y de su génesis: como elemento unitario resultado de la ocupación de un solar vacío y como resultado del proceso de colmatación del interior de una manzana.

${ }^{14}$ El hecho de falta de información contrasta con la aportada para otras tipologías residenciales analizadas a partir de los apeos como las casas y los mesones. 
En las imágenes se puede observar la predominancia del primer tipo de parcela frente al segundo; los corrales de las calles Sol, Peral, Ciegos, Ballestilla, A1var Negro y el corral de las Cañas pertenecen al primer tipo, mientras que, por otro lado, el de las Mozas pertenece al segundo tipo (Figura 2). Este análisis, a priori, desvela que los corrales son el resultado de la necesidad de habitación de un tipo de población creciente mediante la apropiación de solares vacíos y la construcción de aposentos en torno al espacio vacío central. El corral de vecinos puede ser la evolución de la necesidad de habitar esos espacios cerrados y descubiertos por parte de los vecinos de la ciudad, cada vez más densificada.

Las superficies de las parcelas ${ }^{15}$ de los corrales analizados oscilan entre los $247,30 \mathrm{~m}^{2}$ del corral en la calle Sol en la collación de Santa Lucía ${ }^{16}$ y los 784,77 $\mathrm{m}^{2}$ del corral de las Cañas en la calle de los Ángeles en la Magdalena (Tabla 3). La relación entre superficie construida en planta baja y espacios vacíos ha podido ser calculada en solo tres casos debido a la falta de mayores datos por parte de los apeos. Dicha relación se aproxima al 40\%, por lo que se estima que, aunque la presencia del patio central es importante, la superficie edificada sigue siendo predominante ${ }^{17}$.

Tabla 3. Superficies de parcela, porcentaje de vacíos y notas sobre los corrales seleccionados.

\begin{tabular}{|l|c|c|l|}
\hline \multicolumn{1}{|c|}{ Corral } & $\begin{array}{c}\text { Sup. Parcela } \\
\left(\mathrm{m}^{2}\right)\end{array}$ & $\begin{array}{c}\% \\
\text { Vacíos }\end{array}$ & \multicolumn{1}{|c|}{ Notas } \\
\hline En la calle Sol & 247,30 & - & $\begin{array}{l}\text { No se describen los espacios de ma- } \\
\text { nera individual }\end{array}$ \\
\hline De la Morena & 260,77 & $37,06 \%$ & - \\
\hline En la calle de Ciegos & 297,08 & - & $\begin{array}{l}\text { Se ha calculado el área escalando el } \\
\text { dibujo e identificando la parcela en } \\
\text { la calle }\end{array}$ \\
\hline De la Ballestilla & 330,13 & $40,83 \%$ & - \\
\hline
\end{tabular}

${ }^{15}$ La superficie de parcela se ha calculado de manera aproximada aumentando en un $30 \%$ la superficie calculada útil en planta baja para cada uno de los corrales, excepto los corrales de las calles Ciegos y Peral, cuya superficie se ha obtenido mediante el escalado del dibujo de a107 y a115 y su identificación en el parcelario actual.

${ }^{16}$ La referencia de este corral es k102.

${ }^{17}$ La superficie de patio es aún mayor que en la analizada para la casa tipo sevillana, que tenía cerca de un 30\% de espacios descubiertos. NÚÑ̃Z GONZÁLEZ, María: $\mathrm{Ca}$ sas, corrales, mesones y tiendas en la Sevilla del siglo XVI. Arquitectura, dibujo y léxico de alarifes. Tesis inédita. Universidad de Sevilla. Sevilla, 2017, pp. 632; Arquitectura, dibujo y léxico de alarifes en la Sevilla del siglo XVI. Sevilla, 2019 (en prensa). 


\begin{tabular}{|l|c|c|l|}
\hline \multicolumn{1}{|c|}{ Corral } & $\begin{array}{c}\text { Sup. Parcela } \\
\left(\mathrm{m}^{2}\right)\end{array}$ & $\begin{array}{c}\% \\
\text { Vacíos }\end{array}$ & \multicolumn{1}{|c|}{ Notas } \\
\hline De las Mozas & 330,95 & - & Falta la medida del patio \\
\hline En la calle del Peral & 580,91 & - & $\begin{array}{l}\text { Se ha calculado el área escalando el } \\
\text { dibujo e identificando la parcela en } \\
\text { la calle }\end{array}$ \\
\hline $\begin{array}{l}\text { De San Hermenegildo } \\
\text { (Mancebía) }\end{array}$ & 672,18 & - & $\begin{array}{l}\text { Se describen los espacios de ma- } \\
\text { nera conjunta y no permiten el cál- } \\
\text { culo de } \% \text { de vacíos }\end{array}$ \\
\hline $\begin{array}{l}\text { En la barrera de Alvar } \\
\text { Negro }\end{array}$ & 697,95 & $44,36 \%$ & - \\
\hline De las Cañas & 784,77 & - & $\begin{array}{l}\text { Aporta las dimensiones conjuntas } \\
\text { de patio y casapuerta }\end{array}$ \\
\hline Superficie mediana & $330,95 \mathrm{~m}^{2}$ & & \multicolumn{2}{|l}{} \\
\hline
\end{tabular}

\section{Características tipológicas}

En primer lugar, se debe advertir que para el análisis tipológico de los espacios que configuran los corrales se han tenido que descartar los de las calles Peral y Ciegos, por motivos evidentes, ya que la información que aportan las fuentes gráficas se centra en los límites de parcela y no describe sus interiores.

Los corrales, como las casas, tenían una pieza de entrada, la casapuerta, cubierta con tejado a un agua ${ }^{18}$, azotea ${ }^{19}$ o doblada ${ }^{20}$. Sin embargo, no se tienen datos sobre la existencia en ellas de pozos u otro tipo de dotaciones, por lo que puede concluirse que no tenían la misma consideración de espacio multifuncional como ocurre con las casas comunes, sino simplemente como espacio de separación del patio con la calle, de tránsito hacia el interior del corral. Es destacable la ausencia de puerta entre la casapuerta y el patio ${ }^{21}$, en cuyo lugar aparecen huecos de paso que podían resolverse en forma de arco, como ocurre excepcionalmente en el de la barrera de Alvar $\mathrm{Negro}^{22}$.

En los siete casos estudiados, se describen aposentos tanto en planta baja como en planta alta, variando su número según la superficie del solar, desde los

${ }^{18}$ De este tipo eran los k31, k136 y b10v.

19 Con azotea se encuentra el p29r.

${ }^{20}$ Existían dobladas en k102, k31, k136, mozas y b10v.

${ }^{21}$ Puesto que, como ya se ha visto (Núñez González, 2012), en la mayoría de las casas sevillanas existía la puerta de en medio como elemento de seguridad.

${ }^{22}$ Fragmento del apeo del corral en la barrera de Alvar Negro, f523r: "desta dicha pieza se entra por un arco alfizarado a un patio" y "desta casapuerta se entra por un arco apuntalado a un transito". 
cinco que poseía el de la barrera de Alvar Negro hasta los veintiuno de las Mozas o los veintitrés de San Hermenegildo. Según los escasos datos acerca de los aposentos, podían estar doblados al mismo tamaño dando lugar a una planta alta o tejados a un agua. La superficie mediana de los aposentos era de $10,48 \mathrm{~m}^{2}$, variando desde los $7 \mathrm{~m}^{2}$ (b10v) a los $23,29 \mathrm{~m}^{2}(\mathrm{k} 136)^{23}$. Solo se describe una ventana, hacia el patio en un aposento del corral de la Ballestilla (Figura 3).

Los elementos más característicos de los corrales eran los patios o corrales, espacios vacíos resultado de la construcción de habitaciones hacia los muros linderos, y los balcones o corredores que aparecían para la distribución de las habitaciones en plantas altas. El acceso a la planta primera se realizaba en la mayoría de los casos mediante escaleras desde el patio, bien fueran de albañilería (f523r), de piedra o de caja. Excepcionalmente se han encontrado escaleras en una pieza o un tránsito ${ }^{24}$.

En cuanto al abastecimiento de agua, se citan solo cinco pozos en los siete apeos consultados, lo que supone casi las tres cuartas partes de los casos indicando que era muy común pero no siempre contaban con este tipo de suministro. Por otro lado, poseían una zona reservada para la cocina, a veces como un cobertizo en el patio con una chimenea, en el caso de que no tuvieran un espacio dedicado para esa función ${ }^{25}$. Se describen letrinas o servicios en tres de ellos: Mozas, Morena y San Hermenegildo; además de servidumbres para evacuar las aguas residuales $^{26}$. Solo aparece mencionado un sumidero, el del corral de la Ballestilla, en el centro del patio, siendo probable que en esa calle transcurriera un caño o conducto de saneamiento urbano de la ciudad.

Del análisis de los espacios y los volúmenes, se concluye que existían principalmente dos tipos de corrales según la morfología del patio: el primero basado en la génesis compositiva del corral mediante la colocación de un volumen de habitaciones lineal de una sola planta siguiendo las líneas de los linderos dando lugar al patio, y el segundo, más complejo, en el que los volúmenes de habitaciones incorporan una planta alta a la que se accede mediante corredores altos o balcones, que cambian de manera evidente la visión y morfología del espacio central. A través de fotografías históricas se muestran de manera directa la diferencia entre los tipos señalados ${ }^{27}$.

${ }^{23}$ Puntualmente existía un aposento de 48,04 $\mathrm{m}^{2}$ en f523r.

${ }^{24}$ Ambos tipos de escaleras se han encontrado en el apeo del corral de la barrera de Alvar Negro f523r.

${ }^{25}$ Como ocurre en b10v.

${ }^{26}$ Se describen servidumbres en el corral de las Mozas.

${ }^{27}$ MORALES PADRÓN, Francisco (dir. red.): Los corrales de vecinos, ob. cit., fig. 129; y blog Fotos y postales antiguas de Sevilla: http://bit.ly/2nCE1Qi (Consultado 3-4-2017). 


\section{DIBUJO DE LOS CORRALES}

Las descripciones de los corrales de vecinos dadas por los alarifes en los apeos son muy deficientes. Por un lado, carecen de precisión en las dimensiones de los espacios, porque agrupan elementos que funcional y constructivamente son diferentes y, por otro lado, no describen las plantas altas, por lo que se hace todavía más ardua la tarea del dibujo en planta de los mismos. Especialmente parco en detalles es el apeo del corral de las Mozas. Anteriormente mesón, fue convertido en corral de vecindad y dos casas anejas -las llama tres casas de morada- por la nueva propietaria en 1590. La descripción se basa en un listado de aposentos en planta baja con sus respectivas medidas en varas, las dimensiones de la cocina, la escalera alta y los balcones, pero no localiza las piezas ni las vincula unas con otras. A estas dificultades, se une la falta de precisión en la localización de dos de ellos, el de la Mancebía (San Hermenegildo) y el de la Morena (en el barrio de Don Pedro Ponce, collación de San Salvador), con el fin de obtener, al menos la composición de llenos y vacíos.

A pesar de los obstáculos, sí se han podido realizar tres hipótesis en planta de los corrales de la Ballestilla (Figura 3), el de San Hermenegildo (Figura 4) y el de la calle Sol (Figura 5). Como puede observarse en los dibujos, queda evidente la configuración del tipo arquitectónico de corral: un patio o corral alrededor del cual se sitúan habitaciones - unidades de habitación-, con una entrada -la casapuerta posee una función especialmente de seguridad frente a extraños- y que podía tener una o dos plantas. En la casapuerta solía haber un aposento o pieza que, se supone, servía como alojamiento a los caseros, que controlaban el corral. Se accedía a las plantas altas mediante escaleras situadas en las entradas o en el patio, las cuales daban paso a los corredores o balcones que distribuían las estancias. Las instalaciones comunes a los moradores eran el pozo, siempre situado en el patio ${ }^{28}$, la chimenea que servía de cocina -en planta baja fundamentalmente- y la letrina en el corral -podía estar dentro de una casilla en un lugar descentrado-.

\section{CONCLUSIONES}

Por lo que se ha podido deducir de los apeos, los corrales de vecinos se clasificaban según su volumetría en corrales de una o de dos plantas con corredores altos. Si se atiende a la forma de parcela y a la situación con respecto a la manzana, se definirían como resultado de la transformación de una parcela ya construida o de la apropiación de un solar para su edificación con una fachada tradicional a la calle. $\mathrm{O}$ bien serían el resultado de la colmatación del interior de una manzana, con una entrada en forma de adarve o cajón, derivado de la necesidad de

${ }^{28}$ El patio o el corral donde estaban las habitaciones, en esta tipología arquitectónica, son términos sinónimos. 
los vecinos de hacer uso de los espacios vacíos de una ciudad cada vez más densificada.

Esta tipología residencial estaba muy dispersa por toda la ciudad. De los más de veinticinco corrales de vecinos documentados no existe collación que concentre una mayoría de ellos. Esto puede ser expresivo del tipo de población que vivía en ellos, de origen humilde y también transeúntes a la búsqueda de trabajo o de un destino -lo que implicaría una residencia temporal en la ciudad de muy diversa condición social, no siempre humilde o pobre-.

Esta solución habitacional, se desarrollaba en una o dos plantas alrededor del patio o corral, donde se solía situar un pozo y las dotaciones comunes a los vecinos, tales como la cocina con su chimenea y la letrina -bajo un cobertizo-. Su superficie mediana rondaba los $300 \mathrm{~m}^{2}$, una cifra muy inferior a la de corrales hoy conservados en la ciudad, como el del Conde o el de Tromperos.

Este tipo de inmueble ha evolucionado en el tiempo hasta llegar a nuestros días como una tipología de carácter humilde y de clase baja, a veces considerada de manera peyorativa, consideración que no parecía tener en el siglo XVI - pues estaba extendida por toda la ciudad-, aunque en algunos de ellos residieran trabajadores emigrantes, portugueses, montañeses o moriscos liberados y extranjeros en busca de trabajo y acomodo en la ciudad.

\section{APÉNDICE DOCUMENTAL}

\begin{tabular}{|c|c|c|c|c|c|}
\hline $\mathrm{N}$ & Nombre & Collación & Propietario & Año & Referencia \\
\hline 1 & $\begin{array}{l}\text { Salvador } \\
\text { García }\end{array}$ & $\begin{array}{l}\text { Extramu- } \\
\text { ros }\end{array}$ & $\begin{array}{l}\text { Salvador Gar- } \\
\text { cía, clérigo }\end{array}$ & 1595 & $\begin{array}{l}\text { AHPSPN, leg. } 4995 \text {, año } \\
1595 \text {, of. } 7 \text {, fol. } 105\end{array}$ \\
\hline 2 & $\begin{array}{l}\text { De las Ca- } \\
\text { ñas }\end{array}$ & $\begin{array}{l}\text { La Magda- } \\
\text { lena }\end{array}$ & $\begin{array}{l}\text { Hospital del } \\
\text { Cardenal }\end{array}$ & 1580 & $\begin{array}{l}\text { AHDPS, Hospital del Car- } \\
\text { denal, libro 3, apeo } 136\end{array}$ \\
\hline 3 & $\begin{array}{l}\text { En la ba- } \\
\text { rrera de Al- } \\
\text { var Negro }\end{array}$ & $\begin{array}{l}\text { Omnium } \\
\text { Sancto- } \\
\text { rum }\end{array}$ & $\begin{array}{l}\text { Rodrigo } \\
\text { Franco, merca- } \\
\text { der, las dejó a } \\
\text { la Fábrica }\end{array}$ & 1542 & $\begin{array}{l}\text { ACS, Fábrica, libro } 09717 \text {, } \\
\text { fol. } 523 \mathrm{r}-524 \mathrm{r}\end{array}$ \\
\hline 4 & De Gallinas & $\begin{array}{l}\text { Omnium } \\
\text { Sancto- } \\
\text { rum }\end{array}$ & & 1587 & $\begin{array}{l}\text { AHPSPN, of. 1, legajo } 172, \\
\text { años } 1586-1587, \text { fol. } 204\end{array}$ \\
\hline 5 & Corral & $\begin{array}{l}\text { Omnium } \\
\text { Sancto- } \\
\text { rum }\end{array}$ & $\begin{array}{l}\text { Hospital Amor } \\
\text { de Dios }\end{array}$ & 1663 & $\begin{array}{l}\text { AHDPS, Hospital Amor de } \\
\text { Dios, libros 8-bis y 9, apeo } \\
107\end{array}$ \\
\hline 6 & Corral & $\begin{array}{l}\text { Omnium } \\
\text { Sancto- } \\
\text { rum }\end{array}$ & $\begin{array}{l}\text { Hospital Amor } \\
\text { de Dios }\end{array}$ & 1663 & $\begin{array}{l}\text { AHDPS, Hospital Amor de } \\
\text { Dios, libros 8-bis y } 9 \text {, apeo } \\
115\end{array}$ \\
\hline
\end{tabular}




\begin{tabular}{|c|c|c|c|c|c|}
\hline $\mathrm{N}$ & Nombre & Collación & Propietario & Año & Referencia \\
\hline 7 & $\begin{array}{l}\text { De las Pal- } \\
\text { mas }\end{array}$ & $\begin{array}{l}\text { San Barto- } \\
\text { lome }\end{array}$ & & 1597 & $\begin{array}{l}\text { AHPSPN, of. } 6 \text {, año } 1597 \text {, } \\
\text { leg. } 4169 \text {, fol. } 1310\end{array}$ \\
\hline 8 & $\begin{array}{l}\text { En San } \\
\text { Bernardo }\end{array}$ & $\begin{array}{l}\text { San } \\
\text { Bernardo }\end{array}$ & $\begin{array}{l}\text { Doctor Andrés } \\
\text { Çamudio de } \\
\text { Alfaro }\end{array}$ & 1587 & $\begin{array}{l}\text { AHPS, legajo } 11.605 \text {, of. } \\
18 \text {, año } 1587 \text {, libro } 1 \text {, fol. } \\
814 \mathrm{v}-815 \mathrm{v}\end{array}$ \\
\hline 9 & Del Moro & $\begin{array}{l}\text { San } \\
\text { Marcos/ } \\
\text { San } \\
\text { Román }\end{array}$ & $\begin{array}{l}\text { Matrimo- } \\
\text { nio vasco Or- } \\
\text { tuño de Bilbao } \\
\text { la Vieja y Ma- } \\
\text { ría Ochoa, su } \\
\text { mujer }\end{array}$ & 1580 & $\begin{array}{l}\text { AHPSPN, oficio } 15 \text {, año } \\
1580 \text {, fol. } 129 \text {, leg. } 9223\end{array}$ \\
\hline 10 & $\begin{array}{l}\text { De Juan } \\
\text { Guerra }\end{array}$ & $\begin{array}{l}\text { San } \\
\text { Martín }\end{array}$ & $\begin{array}{l}\text { Juan Guerra, } \\
\text { mercader de } \\
\text { azúcar }\end{array}$ & 1585 & $\begin{array}{l}\text { AHDPS, Hospital de las } \\
\text { Bubas, legajo 3-bis, apeo } \\
25 \text {, fol. 22v }\end{array}$ \\
\hline 11 & $\begin{array}{l}\text { De } \\
\text { Toqueros }\end{array}$ & $\begin{array}{l}\text { San } \\
\text { Nicolás }\end{array}$ & & 1563 & $\begin{array}{l}\text { AHPSPN, legajo 4047, ofi- } \\
\text { cio } 6\end{array}$ \\
\hline 12 & $\begin{array}{l}\text { Los } \\
\text { Tromperos }\end{array}$ & $\begin{array}{l}\text { San } \\
\text { Nicolás }\end{array}$ & & 1542 & $\begin{array}{l}\text { ACS, MC, libro 09163, fol. } \\
461 \mathrm{r}\end{array}$ \\
\hline 13 & $\begin{array}{l}\text { Martín } \\
\text { López }\end{array}$ & $\begin{array}{l}\text { San } \\
\text { Salvador }\end{array}$ & $\begin{array}{l}\text { Martín López, } \\
\text { chapinero }\end{array}$ & 1542 & $\begin{array}{l}\text { ACS, MC, libro } 09163 \text {, } \\
\text { fol. } 405 \mathrm{v}\end{array}$ \\
\hline 14 & $\begin{array}{l}\text { De las } \\
\text { Vacas }\end{array}$ & $\begin{array}{l}\text { San } \\
\text { Salvador }\end{array}$ & & 1542 & $\begin{array}{l}\text { ACS, MC, libro } 09163 \text {, } \\
\text { fol. } 434 \mathrm{r}\end{array}$ \\
\hline 15 & $\begin{array}{l}\text { De la } \\
\text { Morena }\end{array}$ & $\begin{array}{l}\text { San } \\
\text { Salvador }\end{array}$ & $\begin{array}{l}\text { Hospital de las } \\
\text { Cinco Llagas }\end{array}$ & 1571 & $\begin{array}{l}\text { AHDPS, Hospital Cinco } \\
\text { Llagas, libro } 1 \text { (1571), } \\
\text { fol. 29r / AHDPS, Hospi- } \\
\text { tal Cinco Llagas, libro } 2 \\
\text { (1578), fol. 114v }\end{array}$ \\
\hline 16 & $\begin{array}{l}\text { De la } \\
\text { Ballestilla }\end{array}$ & $\begin{array}{l}\text { San } \\
\text { Salvador }\end{array}$ & $\begin{array}{l}\text { Hospital de las } \\
\text { Bubas }\end{array}$ & 1585 & $\begin{array}{l}\text { AHDPS, Hospital de las } \\
\text { Bubas, legajo 3-bis apeo } 35 \text {, } \\
\text { fol. 10v }\end{array}$ \\
\hline 17 & $\begin{array}{l}\text { De las } \\
\text { Mozas }\end{array}$ & $\begin{array}{l}\text { San } \\
\text { Salvador }\end{array}$ & $\begin{array}{l}\text { Malgarida } \\
\text { Hernández, } \\
\text { viuda de Fran- } \\
\text { cisco López }\end{array}$ & 1585 & $\begin{array}{l}\text { AHPSPN, legajo } 16740 \text {, } \\
\text { of. } 24 \text {, escritura } 1590 \text {, fol. } \\
459 \mathrm{r}-468 \mathrm{r}\end{array}$ \\
\hline 18 & $\begin{array}{l}\text { Del } \\
\text { Marañuelo }\end{array}$ & $\begin{array}{l}\text { San } \\
\text { Salvador }\end{array}$ & $\begin{array}{l}\text { Doña Ana Se- } \\
\text { garra (hasta } \\
\text { 1544) / Don } \\
\text { Juan Ponce de } \\
\text { León }\end{array}$ & 1544 & - \\
\hline
\end{tabular}




\begin{tabular}{|c|c|c|c|c|c|}
\hline $\mathrm{N}$ & Nombre & Collación & Propietario & Año & Referencia \\
\hline 19 & Del Agua & $\begin{array}{l}\text { Santa Ca- } \\
\text { talina }\end{array}$ & $\begin{array}{l}\text { Hospital de las } \\
\text { Cinco Llagas }\end{array}$ & 1677 & $\begin{array}{l}\text { AHDPS, Hospital Cinco } \\
\text { Llagas, legajo } 73 \text {, protocolo } \\
181\end{array}$ \\
\hline 20 & De calle Sol & $\begin{array}{l}\text { Santa Lu- } \\
\text { cía }\end{array}$ & Hosp. Cardenal & 1580 & $\begin{array}{l}\text { AHDPS, Hospital del Car- } \\
\text { denal, libro 3, apeo } 102\end{array}$ \\
\hline 21 & Corral & $\begin{array}{l}\text { Santa } \mathbf{M}^{\mathrm{a}} \\
\text { la Blanca }\end{array}$ & $\begin{array}{l}\text { Doña Fran- } \\
\text { cisca Rigón, } \\
\text { vende el corral }\end{array}$ & 1597 & $\begin{array}{l}\text { AHPSPN, of. } 6 \text {, año } 1597 \text {, } \\
\text { leg. } 4169 \text {, fol. } 81\end{array}$ \\
\hline 22 & $\begin{array}{l}\text { De Don Pe- } \\
\text { dro Suárez }\end{array}$ & $\begin{array}{l}\text { Santa Ma- } \\
\text { ría la Ma- } \\
\text { yor }\end{array}$ & $\begin{array}{l}\text { Pedro Suárez } \\
\text { de Castilla }\end{array}$ & 1585 & $\begin{array}{l}\text { AHDPS, Hospital de las } \\
\text { Bubas, legajo 3-bis, apeo } \\
50 \text {, fol. 4r }\end{array}$ \\
\hline 23 & $\begin{array}{l}\text { De San Her- } \\
\text { menegildo }\end{array}$ & $\begin{array}{l}\text { Santa Ma- } \\
\text { ría la Ma- } \\
\text { yor }\end{array}$ & $\begin{array}{l}\text { Hospital del } \\
\text { Cardenal }\end{array}$ & 1580 & $\begin{array}{l}\text { AHDPS, Hospital del Car- } \\
\text { denal, libro 3, apeo } 31\end{array}$ \\
\hline 24 & Del Conde & Santiago & - & 1594 & $\begin{array}{l}\text { AHPSPN, of. } 6 \text {, libro } 4155 \text {, } \\
\text { año } 1594 \text {, fol. } 983\end{array}$ \\
\hline 25 & $\begin{array}{l}\text { Calle del } \\
\text { Conde }\end{array}$ & Santiago & $\begin{array}{l}\text { Don Juan de } \\
\text { Vargas y Soto- } \\
\text { mayor, } 24 \text { de } \\
\text { Sevilla }\end{array}$ & 1632 & $\begin{array}{l}\text { AHPSPN, of. } 6 \text {, leg. } 4.341 \text {, } \\
\text { año } 1632 \text {, fol. } 983\end{array}$ \\
\hline 26 & $\begin{array}{l}\text { Calle de la } \\
\text { Cava }\end{array}$ & Triana & $\begin{array}{l}\text { Matrimo- } \\
\text { nio vasco Or- } \\
\text { tuño de Bilbao } \\
\text { la Vieja y Ma- } \\
\text { ría Ochoa, su } \\
\text { mujer }\end{array}$ & 1580 & $\begin{array}{l}\text { AHPSPN, oficio } 15 \text {, año } \\
1580 \text {, fol. } 129 \text {, leg. } 9223\end{array}$ \\
\hline 27 & De la Parra & Triana & - & 1589 & $\begin{array}{l}\text { AHPSPN, legajo } 16739 \text {, } \\
\text { año } 1589 \text {, of. } 24 \text {, libro } 2 \text {, fol. } \\
611 \mathrm{v}\end{array}$ \\
\hline 28 & $\begin{array}{l}\text { De los } \\
\text { Olmos }\end{array}$ & - & - & 1543 & $\begin{array}{l}\text { AHPSPN, legajo 3353, re- } \\
\text { gistro } 14\end{array}$ \\
\hline
\end{tabular}

Fecha de recepción: 25 de octubre de 2018

Fecha de aceptación: 5 de febrero de 2019 


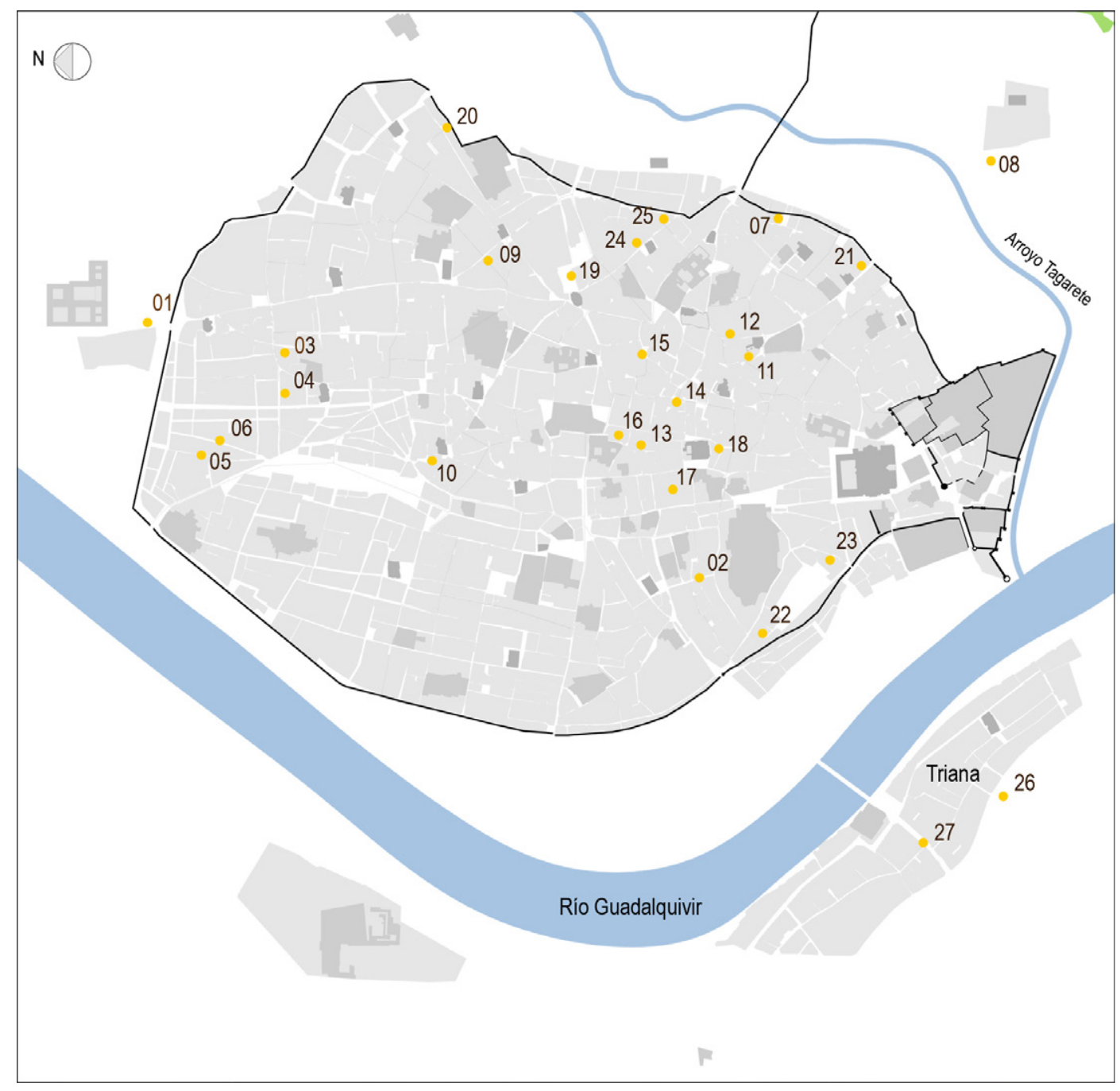

Figura 1. Plano de situación aproximada de los corrales referenciados. 01. Salvador García, 02. De las Cañas, 03. De Alvar Negro, 04. De Gallinas, 05. Ciegos, 06. Peral, 07. Las Palmas (San Bartolomé), 08. San Bernardo, 09. Del Moro, 10. Juan Guerra, 11. De Toqueros, 12. Los Tromperos, 13. Martín López, 14. De las Vacas, 15. De la Morena, 16. Ballestilla, 17. De las Mozas, 18. Del Marañuelo, 19. Del Agua, 20. De la calle Sol, 21. De Santa María la Blanca, 22. De Don Pedro Suárez, 23. San Hermenegildo, 24. Del Conde, 25. De la calle del Conde, 26. De la calle de la Cava, 27. De la Parra (Triana).

Plano: María Núñez González. 


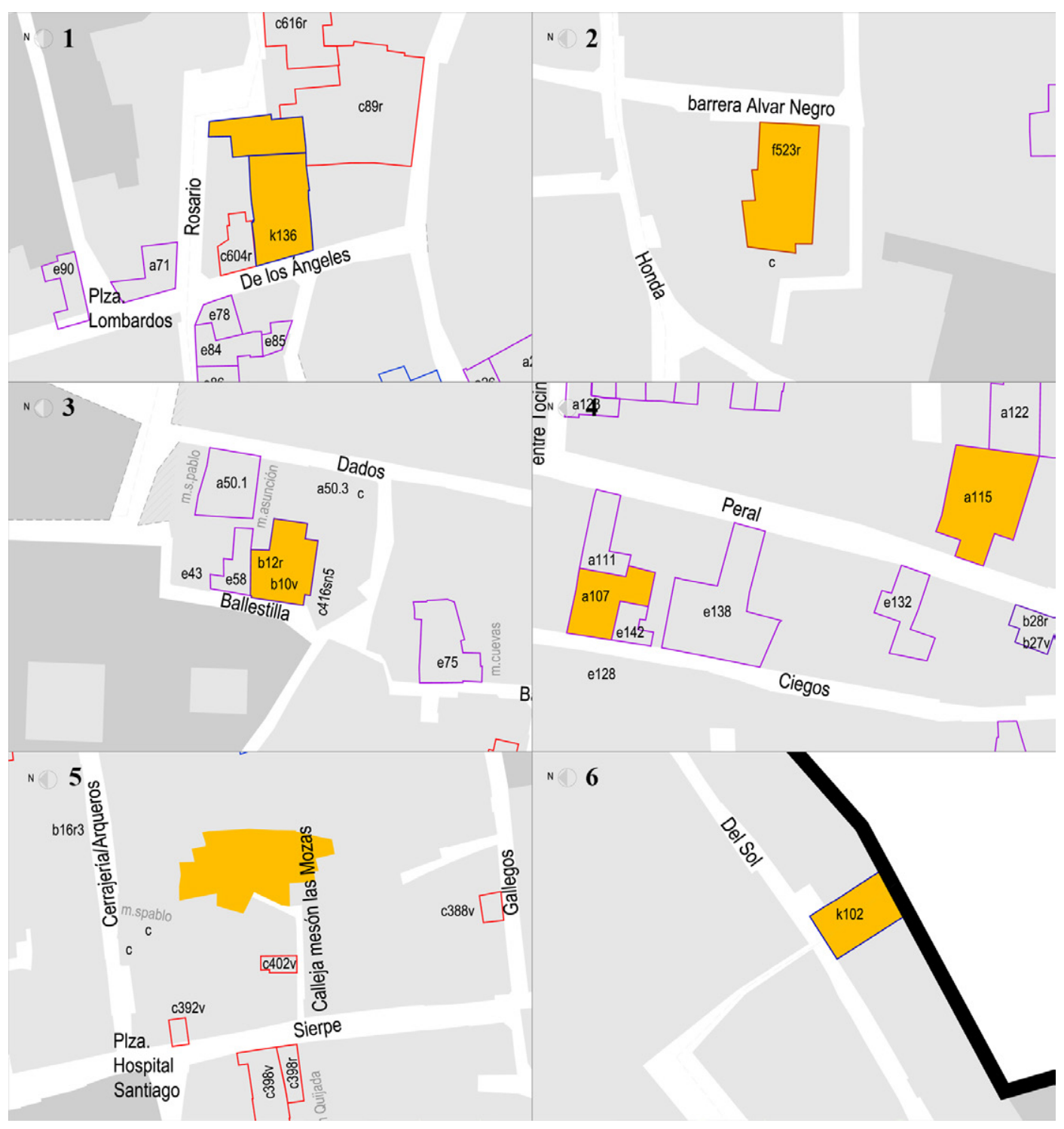

Figura 2. Relación de las parcelas de los corrales respecto a la manzana urbana. 1: corral de las Cañas; 2: corral de la Barrera de Alvar Negro; 3: corrales de las calles Peral y Ciegos; 4: corral de la Ballestilla; 5: corral de las Mozas; 6: corral de la calle Sol. Plano: María Núñez González. 


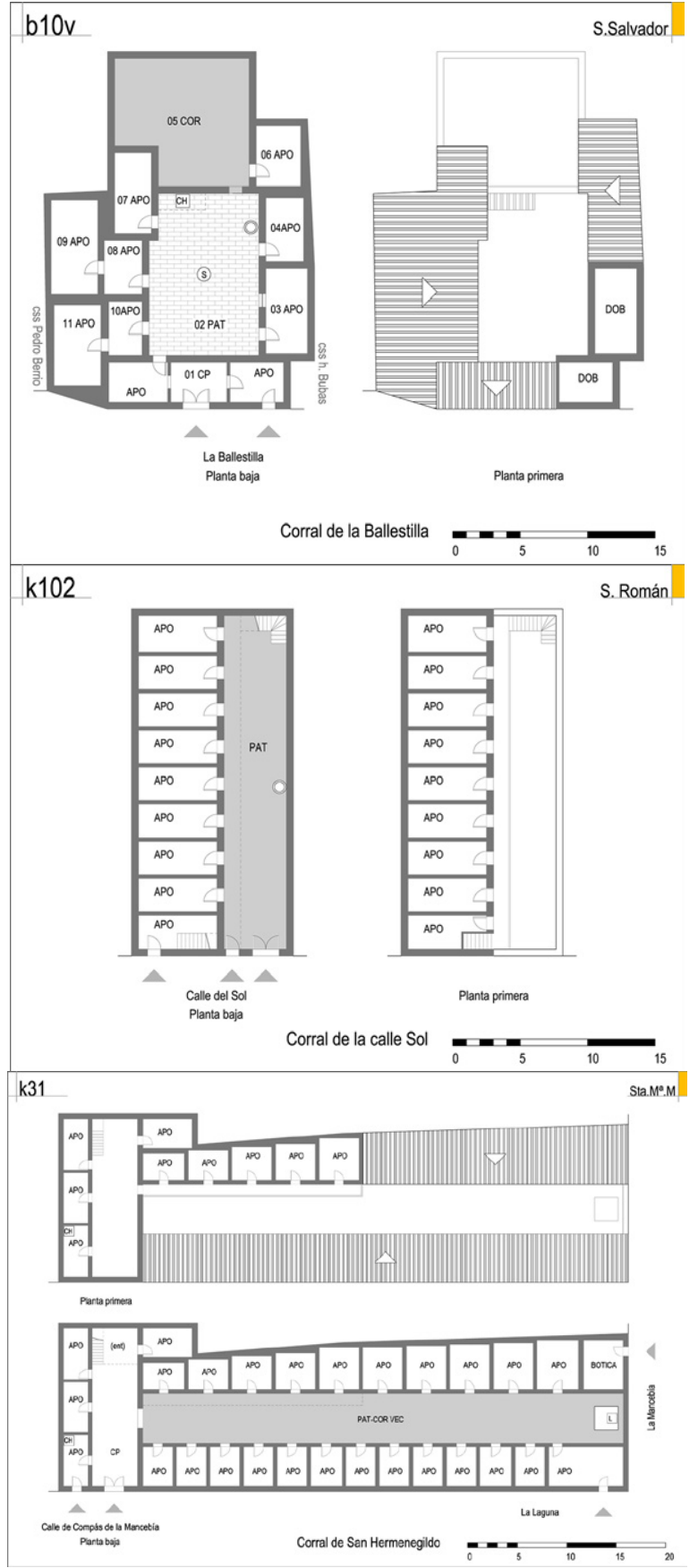

Figura 3. Dibujo del corral de la Ballestilla en la collación de San Salvador, en la Ballestilla. Este corral de vecinos tenía principalmente una planta de altura, un patio solado y un corral, alrededor de los cuales se situaban los aposentos. Además, contaba con un sumidero, una chimenea bajo un tejadillo y un pozo en el patio. Plano: María Núñez González. Escala gráfica en varas castellanas.

Figura 4. Dibujo del corral de la calle Sol (k102) en San Román. Tenía dos plantas de altura, tres puertas a la calle -dos al patio y una al primer aposento de donde partía una de las escaleras para la planta alta- y un patio solado de rebocado que tenía un corredor y un pozo. Plano: María Núñez González. Escala gráfica en varas castellanas.

Figura 5. Dibujo del corral de San Hermenegildo en la Mancebía, en Santa María la Mayor. Tenía una planta de altura, salvo en la zona de la entrada que tenía dos. Destaca la entrada al patio sin puertas y la existencia de un cobertizo para la letrina al fondo de este. Además, albergaba una botica (cuarto de prostitutas). Plano: María Núñez González Escala gráfica en varas castellanas. 